\title{
Importance of genotyping patients for HLA-C*06:02: it provides not only pharmacogenetics implication in response to biologics drugs but also drug survival and drug-related costs information
}

\author{
Marina Talamonti ${ }^{1,2 \#}$, Marco Galluzzo ${ }^{1,3 \#}$ \\ ${ }^{1}$ Dermatology Unit, Fondazione Policlinico Tor Vergata, Rome, Italy; ${ }^{2}$ Department of Systems Medicine, ${ }^{3}$ Department of Experimental Medicine, \\ University of Rome "Tor Vergata”, Rome, Italy \\ "These authors contributed equally to this work. \\ Correspondence to: Marina Talamonti. Department of Systems Medicine, University of Rome "Tor Vergata", Viale Oxford 81, 00133 Rome, Italy. \\ Email: talamonti.marina@gmail.com. \\ Provenance and Peer Review: This article was commissioned and reviewed by the Academic Editor Dr. Weijun Jiang (Nanjing Normal University, \\ Department of Reproductive and Genetics, Institute of Laboratory Medicine, Jinling Hospital, Nanjing University School of Medicine, Nanjing, \\ China). \\ Comment on: van Vugt LJ, van den Reek JMPA, Hannink G, et al. Association of HLA-C*06:02 Status With Differential Response to Ustekinumab in \\ Patients With Psoriasis: A Systematic Review and Meta-analysis. JAMA Dermatol 2019;155:708-15.
}

Submitted Feb 15, 2020. Accepted for publication Mar 18, 2020.

doi: $10.21037 /$ atm.2020.04.05

View this article at: http://dx.doi.org/10.21037/atm.2020.04.05

Current psoriasis treatments have limited efficacy due to variations among individuals. With advances in GWAS, researchers have made progress studying how genetic variation of genomic loci may affect phenotypic variability.

Such associations help to predict risk for intervention strategies and targeted prevention as well as providing indications for the development of new drugs.

In several pharmacogenetic studies, variants in specific genes have been shown to be associated with therapy clinical response. The effects of these variants can be applied to indicate optimal therapy, reduce drug events adverse and expenditure costs related.

In recent years, our group, in multiple studies and even in multicenter analyses (national and international) with different patient groups and different case numbers, has found a positive correlation between presence of HLA-C ${ }^{*} 06: 02$ allele and response to ustekinumab in shortand long-term outcomes [104 weeks of observation (1-4)].

This result was demonstrated with similar or less significance by other groups with some exceptions of mixed results (5-8).

A recent systematic review and meta-analysis conducted by van Vugt et al. regarding these published data, showed a differential response to ustekinumab therapy based on
HLA-C*06:02 status in patients with psoriasis. Although $\mathrm{C}^{*} 06: 02$-positive (HLA-C*06:02-POS) patients had high response rates at 6 months, response rates were also high in the HLA-C*06:02-negative (HLA-C*06:02-NEG) group. Therefore, the findings suggest that patients should not be excluded from ustekinumab treatment based on a negative HLA-C*06:02 status. Furthermore, the authors declared: "beterogeneity complicated generalizability and thus implementation of our findings, which may probibit the current use of HLA-C*06:02 as a single predictor for ustekinumab treatment response in patients with psoriasis in clinical practice" (9).

First, in our previous studies we never concluded that HLA-C*06:02-NEG patients have no chance of response to the drug. We have only highlighted the predictivity of one of the alleles that most associate with psoriatic disease in response to ustekinumab.

Second, we are also firmly convinced that realizing personalized treatment in psoriasis care may depend on finding a set of biomarkers, rather than a single marker, that in combination are strongly predictive of therapeutic response, as van Vugt et al. concluded in their work.

More recently the BADBIR Study Group (the British Association of Dermatologists Biologic and Immunomodulators Register) the BSTOP Study Group 
(the Biomarkers of Systemic Treatment Outcomes in Psoriasis), and the PSORT Consortium conducted a study to assess association of HLA-C ${ }^{*}$ 06:02 status and response to adalimumab and ustekinumab. One thousand three hundred twenty-six participants were included in the final analysis. As the authors confirmed, while size heterogeneity across time points (3, 6 and 12 months) does have some effect, HLA-C ${ }^{*} 06: 02$ is associated with a response to each drug individually. Its response to ustekinumab is better, which is consistent with our observations, and it has a poorer response to adalimumab, which has not previously been established (10).

Furthermore, we recently made an 8-year real-life study on the use of ustekinumab for the treatment of moderateto-severe plaque psoriasis in a cohort of 378 patients. We showed that the presence of allele HLA-C ${ }^{*} 06: 02$ correlates not only with a better response in terms of achieving PASI75, PASI90 and PASI100, but it is also a predictive factor for drug survival.

In observational studies, drug survival may be considered to reliably indicate the overall effectiveness of treatment, since it is mainly determined by the efficacy and safety profile of the drug. Two large registries-PSOLAR and DERMBIO $(11,12)$ - have been used to evaluate the drug survival of ustekinumab for the treatment of chronic moderate-to-severe plaque psoriasis. Recent meta-analyses have also shown that ustekinumab presents higher drugsurvival rates than other biologics in this setting $(13,14)$.

As well, the long-term drug survival of ustekinumab has not been evaluated in many real-life studies.

The purpose of our study was to evaluate efficacy, safety and drug survival of ustekinumab in a cohort of patients with moderate to severe plaque psoriasis.

The period of study in a real-world setting was 8 years. We found a mean retention rate of 3.7 years (95\% CI: $3.3-4.1)$ in HLA-C ${ }^{*} 06: 02-P O S$ patients, compared to 3.3 years (95\% CI: $2.9-3.6)$ in HLA-C ${ }^{*} 06: 02-N E G$ patients $(\mathrm{P}=0.005)$. For HLA-C*06:02-POS patients, the retention rate was $71 \%$ after 5 years, compared to $51 \%$ in HLA$\mathrm{C}^{*} 06: 02-\mathrm{NEG}$ patients.

The role of the HLA-C*06:02 allele was highlighted again in a drop-out analysis. Among $\mathrm{C}^{*}$ 06:02-negative patients, a drop-out rate of $75 \%$ for primary inefficacy occurred, compared to $25 \%$ of $\mathrm{C}^{*} 06: 02$-POS patients. The loss of efficacy recorded was also more common for $\mathrm{C}^{\star} 06: 02-\mathrm{NEG}$ patients $\left(59.5 \%\right.$ ) compared to $\mathrm{C}^{*} 06$ :02-POS patients [40.5\% (15)].

Therefore, we can say that this allele is also a predictor of drug-related costs. Starting the right therapy for the right patient, a therapy that maintains efficacy even in the long-term, reduces the costs related to biological drugs, as well as costs related to the therapeutic switch and new drug inductions.

Psoriasis is an inflammatory skin disease driven by TNF-a/IL-23/IL-17A; both environmental and genetic factors contribute to its pathogenesis, its appearance and severity. Although the antigenic properties leading to the development of psoriasis are still uncertain, some possible candidate molecules include auto-antigens such as keratin 17, ADAMTSL5, LL-37, and neolipids. T cells autoreactive to such self-antigens are restricted in part to HLA-C*06:02 or CD1a. As well, autoreactive T cells evidently include IL17A-producing $\mathrm{T}$ cell clones. In one case in particular, IL17 production may possibly occur very early in the postpresentation phases of the antigen, not necessarily under the mandatory influence of IL-23. Considering the varied plasticity of Th17 cells, in autoimmunity, primed Th17 cells may differentiate into other types of helper $\mathrm{T}$ cells in different cytokine milieu. Additional researches are needed to elucidate the specific role of these auto-antigens in pathophysiology of psoriasis (16).

HLA-C*06:02 allele has been identified as the strongest susceptibility allele for psoriasis in world's population, including Europeans and South Asians. Application of the HLA imputation method for fine-mapping has identified other several variants of HLA class I and II that are less obviously associated yet confer possibility to develop psoriasis. In addition to HLA-C*06:02, psoriasis is associated with HLA-C ${ }^{*} 07: 01$, HLA-C $\mathrm{C}^{*} 07: 02$, HLA-C*07:04, HLA-C*12:03, HLA-B*27, and HLA-B*57.

Nevertheless, the molecular mechanism is still not entirely understood. The question remains how and why HLA-C ${ }^{*} 06: 02$ and a T-cell autoantigen such as LL-37 or ADAMTSL5 can lead to psoriasis while the same is not true of other close HLA-C alleles and peptides. The HLA-C pocket, i.e., the antigen-binding site of HLA molecules, could explain the difference in this respect. Intensive contacts of more hydrogen bonds and salt bridges must be formed between HLA-C*06:02 and its auto-antigen peptide. The HLA-C*06:02-presented auto-antigen peptide must also have the excellent accessible surface and contact area, that contribute to its easy recognition by several TCRs, leading to greater possibilities evoking an immune response. An acceptor sites diversity implies that different HLA molecules can select different peptide repertoires for presentation, although the binding specificities may overlap 
$(17-20)$.

In this sense, it is very likely that HLA-C*06:02-POS and HLA-C ${ }^{*}$ 06:02-NEG status of psoriatic patients identifies different endotypes with biological implications relevant.

This new vision of the role of HLA is in line with recent studies published in the literature. Indeed, a recent study demonstrated that in the Chinese population there are HLA differences in patients with erythrodermic psoriasis compared to those with plaque psoriasis (21).

Some cases of psoriasis still unfortunately do not respond, do not respond adequately or lose response to biotechnological therapies, available to help control psoriasis. The common solution is to switch to other biological drugs, with a corresponding increase in pharmaceutical costs. Genetic polymorphisms have also recently been shown to influence the response of psoriasis patients to biological drugs.

A key goal of the future lies in personalized medicine, using individual genetic profiles to characterize type of psoriasis, the course of the disease, and the subsets of patients most likely to respond to particular drugs.

Therefore, deep knowledge of the psoriasis pathogenesis and the mechanism underlying treatment response can be gained by further larger studies regarding the genetic and immunologic characterization about HLA-C*06:02 status.

\section{Acknowledgments}

Funding: None.

\section{Footnote}

Conflicts of Interest: Both authors have completed the ICMJE uniform disclosure form (available at http://dx.doi. org/10.21037/atm.2020.04.05). The authors have no conflicts of interest to declare.

Ethical Statement: The authors are accountable for all aspects of the work in ensuring that questions related to the accuracy or integrity of any part of the work are appropriately investigated and resolved.

Open Access Statement: This is an Open Access article distributed in accordance with the Creative Commons Attribution-NonCommercial-NoDerivs 4.0 International License (CC BY-NC-ND 4.0), which permits the noncommercial replication and distribution of the article with the strict proviso that no changes or edits are made and the original work is properly cited (including links to both the formal publication through the relevant DOI and the license). See: https://creativecommons.org/licenses/by-nc-nd/4.0/.

\section{References}

1. Talamonti M, Botti E, Galluzzo M, et al. Pharmacogenetics of psoriasis: HLA-Cw6but not LCE3B/3C deletion nor TNFAIP3 polymorphism predisposes to clinical response to interleukin 12/23 blocker ustekinumab. Br J Dermatol 2013;169:458-63.

2. Talamonti M, Galluzzo M, Chimenti S, et al. HLA-C*06 and response to ustekinumab in Caucasian patients with psoriasis: outcome and long-term follow-up. J Am Acad Dermatol 2016;74:374-5.

3. Galluzzo M, Boca AN, Botti E, et al. IL12B (p40) gene polymorphisms contribute to ustekinumab response prediction in psoriasis. Dermatology 2016;232:230-6.

4. Talamonti M, Galluzzo M, van den Reek JM, et al. Role of the HLA-C*06 allele in clinical response to ustekinumab: evidence from real life in a large cohort of European patients. Br J Dermatol 2017;177:489-96.

5. Chiu HY, Wang TS, Chan CC, et al. Human leucocyte antigen-Cw6as a predictor for clinical response to ustekinumab, an interleukin-12/23 blocker, in Chinese patients with psoriasis: a retrospective analysis. $\mathrm{Br} \mathrm{J}$ Dermatol 2014;171:1181-8.

6. Li K, Huang CC, Randazzo B, et al. HLA-C*06:02 allele and response to IL-12/23 inhibition: results from the ustekinumab phase 3 psoriasis program. J Invest Dermatol 2016;136:2364-71.

7. Raposo I, Carvalho C, Bettencourt A, et al. Psoriasis pharmacogenetics: HLA-Cw*0602 as a marker of therapeutic response to ustekinumab. Eur J Dermatol 2017;27:528-30.

8. Anzengruber F, Ghosh A, Maul JT, et al. Limited clinical utility of HLA-Cw6genotyping for outcome prediction in psoriasis patients under ustekinumab therapy: a monocentric, retrospective analysis. Psoriasis (Auckl) 2018;8:7-11.

9. van Vugt LJ, van den Reek JMPA, Hannink G, et al. Association of HLA-C*06:02 Status With Differential Response to Ustekinumab in Patients With Psoriasis: A Systematic Review and Meta-analysis. JAMA Dermatol 2019;155:708-15.

10. Dand N, Duckworth M, Baudry D, et al. HLA-C*06:02 genotype is a predictive biomarker of biologic treatment response in psoriasis. J Allergy Clin Immunol 
2019; 143:2120-30.

11. Strober BE, Bissonnette R, Fiorentino D, et al. Comparative effectiveness of biologic agents for the treatment of psoriasis in a real-world setting: results from a large, prospective, observational study (Psoriasis Longitudinal Assessment and Registry [PSOLAR]). J Am Acad Dermatol 2016;74:851-61.e4.

12. Egeberg A, Iversen L, Gniadecki R, et al. Characteristics of patients receiving ustekinumab compared with secukinumab for treatment of moderate-to-severe plaque psoriasis - nationwide results from the DERMBIO registry. J Eur Acad Dermatol Venereol JEADV 2017;31:1183-7.

13. Lin PT, Wang SH, Chi CC. Drug survival of biologics in treating psoriasis: a meta-analysis of real-world evidence. Sci Rep 2018;8:16068.

14. No DJ, Inkeles MS, Amin M, et al. Drug survival of biologic treatments in psoriasis: a systematic review. J Dermatolog Treat 2018;29:460-6.

15. Galluzzo M, D'Adamio S, Silvaggio D, et al. Ustekinumab treatment for moderate-to-severe plaque psoriasis: eight-year real-life experience. Expert Opin Biol Ther 2020;20:95-104.

Cite this article as: Talamonti $M$, Galluzzo M. Importance of genotyping patients for HLA-C*06:02: it provides not only pharmacogenetics implication in response to biologics drugs but also drug survival and drug-related costs information. Ann Transl Med 2020;8(15):971. doi: 10.21037/atm.2020.04.05
16. Furue M, Kadono T. The contribution of IL-17 to the development of autoimmunity in psoriasis. Innate Immun 2019;25:337-43.

17. Falk K, Rotzschke O, Stevanovic S, et al. Allele-specific motifs revealed by sequencing of self-peptides eluted from MHC molecules. Nature 1991;351:290-6.

18. Falk K, Rotzschke O, Stevanovic S, et al. Pool sequencing of natural HLA-DR, DQ, and DP ligands reveals detailed peptide motifs, constraints of processing, and general rules. Immunogenetics 1994;39:230-42.

19. Ou D, Mitchell LA, Tingle AJ. HLA-DR restrictive supertypes dominate promiscuous $\mathrm{T}$ cell recognition: association of multiple HLA-DR molecules with susceptibility to autoimmune diseases. J Rheumatol 1997;24:253-61.

20. Sette A, Sidney J. Nine major HLA class I supertypes account for the vast preponderance of HLA-A and -B polymorphism. Immunogenetics 1999;50:201-12.

21. Lo Y, Chiu HY, Tsai TF. Clinical Features and Genetic Polymorphism in Chinese Patients with Erythrodermic Psoriasis in a Single Dermatologic Clinic. Mol Diagn Ther 2020;24:85-93. 